\title{
Implicit stomer cowell k-step hybrid block methods for solution of first order ordinary differential equations (ODEs)
}

\author{
AM Badmus ${ }^{1 *}$, YA Yahaya ${ }^{2}$, AO Subair ${ }^{3}$ \\ ${ }^{1}$ Mathematics Department, Nigerian Defence Academy Kaduna, Nigeria \\ ${ }^{2}$ Mathematics and Statistics Department, Federal University of Technology Minna. Nigeria \\ *Corresponding author E-mail: ambadmus@nda.edu.ng
}

Copyright $\odot 2014$ Am Badmus et al. This is an open access article distributed under the Creative Commons Attribution License, which permits unrestricted use, distribution, and reproduction in any medium, provided the original work is properly cited.

\begin{abstract}
This paper focuses on derivation of two implicit hybrid Block methods at $k=3$ and one method at $k=4$. Each block method produced at $k=3$ yielded uniform order 5 and 7 respectively, while the Block implicit method at $\mathrm{k}=4$ produced uniform order 9 . The block analysis of all the methods derived shows that they are zero stable, consistent and convergent. The superiority of these methods over some existing one were demonstrated numerically.
\end{abstract}

Keywords: Consistent And Convergent, Implicit Block Methods, Uniform Order and Zero Stable.

\section{Introduction}

Many life and physical problems can be modeled into differential equation of the form

$y^{\prime}=f(x, y)$

The equation (1.0) occurs in field of sciences and engineering when we come across physical and natural phenomena which, when represented by mathematical models, happen to be differential equations. Some of these differential equations do not possess the closed form solution hence the numerical computation which is the area of mathematics and computer science that creates analyses and implements algorithms for numerical or approximate solutions is adopted to obtain the solution of (1.0).

Many Researchers have worked extensively in this area such as Awoyemi [1], Badmus and Mshelia ([2],[3]), Badmus and Adegboye [4],Badmus et al [5], Odekunle et al ([7],[8], Yahaya and Adegboye [10], to mention but a few.

The aim of this research paper is to develop a high order, zero stable and consistent block method at various values of $\mathrm{k}$ and use it to solve some existing known problems to ascertain the level of convergence.

Definition 1.0: One-Step Method

The construct an approximate solution $x_{(k+1)}=x(\mathrm{t})_{\mathrm{k}}$, using only one previous approximation $x_{\mathrm{k}}$. The approach in this method enjoys the virtue that the step size (h) can be changed at every iteration, if desired, thus providing a mechanism for error control.

A general expression of one-step method is $y_{n+1}=y_{n}+h f\left(x_{n}, y_{n}\right)$ where $f\left(x_{n}, \mathrm{y}_{\mathrm{n}}, \mathrm{h}\right)=\mathrm{f}_{\mathrm{n}}=f\left(x_{n}, \mathrm{y}_{\mathrm{n}}\right)$

(Lambert [6])

Definition 1.1: Stomer Cowell Method

A linear Multistep Method of (1.2) is said to be Stomer Cowell if and only if

$\sum_{\mathrm{j}=0}^{\mathrm{t} m-1} \alpha_{\mathrm{j}}=0$

Definition 1.2: Linear Multistep Method (LMM)

If a computational method for determining a sequence between $\left[y_{n}\right]$ takes the form of a linear relationship betweeny ${ }_{n+j}, f_{n+j}, j=0,1,2, \ldots, k$, then we call it a LMM of step number $K$ or a linear k-step method.

A linear $\mathrm{k}$-step method is mathematically defined as 
$a_{k} y_{n+k}+a_{k-1} y_{n+k-1} \ldots \ldots \ldots+a_{1} y_{n+1}+a_{o} y_{n}=h\left(\beta_{k} f_{n+k}+\ldots \ldots \ldots+\beta_{1} f_{n+1}+\beta_{o} f_{n}\right)$ Which can be written in compartment form as?

$\sum_{\mathrm{j}=0}^{\mathrm{k}} \alpha_{\mathrm{j}} \mathrm{y}_{\mathrm{n}+\mathrm{j}}=\mathrm{h} \sum_{\mathrm{j}=0}^{\mathrm{k}} \beta_{\mathrm{j}} \mathrm{f}_{\mathrm{n}+\mathrm{j}}$

Where $\left|\alpha_{\mathrm{k}}\right|+\left|\beta_{\mathrm{k}}\right| \neq 0$ and $\beta_{\mathrm{k}}=1$ when $\beta_{\mathrm{k}} \neq 0$, the scheme becomes an implicit scheme, otherwise explicit scheme.

Subair [9]

Definition 1.3: Zero Stability

The linear multistep method (1.2) is said to satisfy the root conditions if all the roots of the first characteristics polynomial have modulus less than or equal to unity and those of modulus unity are simple. The method (1.2) is said to be zero stable if it satisfies the root condition Lambert [6].

Theorem 1.0: Fundamental Theorem of Dahlquist.

The necessary and sufficient conditions for a Linear Multistep Method (LMM) to be convergent are that it must be Consistent and Zero stable.

\section{Theorem 1.1: Dalhquist Order Barrier for LMM.}

a) A Zero-stable, K-step LMM is maximum order P with $\mathrm{p}=\left(\begin{array}{c}\mathrm{k}+1, \text { for } \mathrm{k} \text { odd } \\ \mathrm{k}+2 \text {,for k even }\end{array}\right)$

b) An explicit LMM cannot attain A-stability if the step number, $\mathrm{k}$ is suck that

c) $\mathrm{k}>2$

d) The Order P of an A-stable LMM cannot exceed two. In fact, the Trapezoidal rule which is of Order P = 2 with step number, $\mathrm{k}=1$ known for its A- stability has the smallest Error constant of $\mathrm{C}^{*}=\frac{1}{12}$ Lambert [6].

\section{Methodology}

Given a power series of the form

$\mathrm{p}(\mathrm{x})=\sum_{\mathrm{j}=0}^{\infty} \alpha_{\mathrm{j}} \mathrm{x}^{\mathrm{j}}$

Which is used as our basis to produce an approximate solution to (1.0) as?

$\mathrm{y}(\mathrm{x})=\sum_{\mathrm{j}=0}^{\mathrm{s}+\mathrm{t}-1} \alpha_{\mathrm{j}} \mathrm{x}^{\mathrm{j}}$

and

$\mathrm{y}^{\prime}(\mathrm{x})=\sum_{\mathrm{j}=0}^{\mathrm{s}+\mathrm{t}-1} \mathrm{j} \alpha_{\mathrm{j}} \mathrm{x}^{\mathrm{j}}=\mathrm{f}(\mathrm{x}, \mathrm{y})$

Where $\alpha_{j}{ }^{\prime}$ s are the parameters to be determined, $s$ and $t$ are the points of collocation and interpolation respectively. This process leads to $(\mathrm{s}+\mathrm{t}-1)$ non-linear system of equations with $(\mathrm{s}+\mathrm{t}-1)$ unknown coefficients, which are to be determined by the use of maple 17 mathematical software.

\subsection{Hybrid Block methods derived at $k=3$}

a) Using equations (2.1) and (2.2), $\mathrm{s}=3, \mathrm{t}=3$. our choice of degree of polynomial is $(\mathrm{s}+\mathrm{t}-1)$. Equations (2.1) and (2.2) are interpolated and collocated respectively at the points $x=\left\{x_{n+\frac{1}{2}}, x_{n+\frac{3}{2}}, x_{n+\frac{5}{2}}\right)$ which gives the following non-linear system of equations of the form

$\sum_{\mathrm{j}=0}^{\mathrm{s}+\mathrm{t}-1} \alpha_{\mathrm{j}} \mathrm{x}_{\mathrm{n}+\mathrm{i}}^{\mathrm{j}}=\mathrm{y}_{\mathrm{n}+\mathrm{i}}$

$\sum_{j=1}^{s+t-1} j \alpha_{j} x_{n+i}^{j-1}=f_{n+i} \quad$ where $i=\left(\frac{1}{2}, \frac{3}{2}, \frac{5}{2}\right)$

With the mathematical software, we obtain the continuous formulation of equations (2.3) and (2.4) as follows

$y(x)=\left[-\frac{225}{128}+\frac{915}{64 h}\left(x-x_{n}\right)-\frac{407}{16 h^{2}}\left(x-x_{n}\right)^{2}+\frac{149}{8 h^{3}}\left(x-x_{n}\right)^{3}-\frac{49}{8 h^{4}}\left(x-x_{n}\right)^{4}+\frac{3}{4 h^{5}}\left(x-x_{n}\right)^{5}\right] y_{n+\frac{1}{2}} \cdot+\left[\frac{25}{16}-\right.$

$152 h x-x n+232 h 2 x-x n 2-6 h 3 x-x n 3+1 h 4 x-x n 4 y n+32$

$+\left[\frac{153}{128}-\frac{435}{64 h}\left(x-x_{n}\right)+\frac{223}{16 h^{2}}\left(x-x_{n}\right)^{2}-\frac{101}{8 h^{3}}\left(x-x_{n}\right)^{3}+\frac{41}{8 h^{4}}\left(x-x_{n}\right)^{4}-\frac{3}{4 h^{5}}\left(x-x_{n}\right)^{5}\right] y_{n+\frac{5}{2}}$

$+\left[-\frac{225 h}{128}+\frac{465}{64}\left(x-x_{n}\right)-\frac{167}{16 h}\left(x-x_{n}\right)^{2}+\frac{55}{8 h^{2}}\left(x-x_{n}\right)^{3}-\frac{17}{8 h^{3}}\left(x-x_{n}\right)^{4}+\frac{1}{4 h^{4}}\left(x-x_{n}\right)^{5}\right] f_{n+\frac{1}{2}}$

$+\left[-\frac{75 h}{32}+\frac{205}{16}\left(x-x_{n}\right)-\frac{99}{4 h}\left(x-x_{n}\right)^{2}+\frac{41}{2 h^{2}}\left(x-x_{n}\right)^{3}-\frac{15}{2 h^{3}}\left(x-x_{n}\right)^{4}+\frac{1}{h^{4}}\left(x-x_{n}\right)^{5}\right] f_{n+\frac{3}{2}}$

$+\left[-\frac{45 h}{128}+\frac{129}{64}\left(x-x_{n}\right)-\frac{67}{16 h}\left(x-x_{n}\right)^{2}+\frac{31}{8 h^{2}}\left(x-x_{n}\right)^{3}-\frac{13}{8 h^{3}}\left(x-x_{n}\right)^{4}+\frac{1}{4 h^{4}}\left(x-x_{n}\right)^{5}\right] f_{n+\frac{5}{2}}$

When equation (2.5) evaluated at $x=x_{n+j}$ where $j=0,1,2$ and its first derivative also evaluated at $x=x_{n+j}, j=0,2$ gives the following set of discrete schemes to form the first hybrid block method at $\mathrm{k}=3$.

$\frac{153}{128} \mathrm{y}_{\mathrm{n}+\frac{5}{2}}+\frac{25}{16} \mathrm{y}_{\mathrm{n}+\frac{3}{2}}-\frac{225}{128} \mathrm{y}_{\mathrm{n}+\frac{1}{2}}-\mathrm{y}_{\mathrm{n}}=\frac{45}{128} \mathrm{hf}_{\mathrm{n}+\frac{5}{2}}+\frac{75}{32} \mathrm{hf}_{\mathrm{n}+\frac{1}{2}}+\frac{225}{128} \mathrm{hf}_{\mathrm{n}+\frac{3}{2}}$ 
$\frac{11}{128} y_{n+\frac{5}{2}}+\frac{9}{16} y_{n+\frac{3}{2}}-y_{n+1}+\frac{45}{128} y_{n+\frac{1}{2}}=\frac{3}{128} h_{n+\frac{5}{2}}+\frac{9}{32} h f_{n+\frac{3}{2}}-\frac{9}{128} h f_{n+\frac{1}{2}}$

$\frac{45}{128} y_{n+\frac{5}{2}}-y_{n+2}+\frac{9}{16} y_{n+\frac{3}{2}}+\frac{11}{128} y_{n+\frac{1}{2}}=\frac{9}{128} h_{n+\frac{5}{2}}-\frac{9}{32} h_{n+\frac{3}{2}}-\frac{3}{128} h_{n+\frac{1}{2}}$

$435 y_{n+\frac{5}{2}}+480 y_{n+\frac{3}{2}}-915 y_{n+\frac{1}{2}}=129 h_{n+\frac{5}{2}}+820 h f_{n+\frac{3}{2}}+465 h f_{n+\frac{1}{2}}-64 h f_{n}$

$93 y_{n+\frac{5}{2}}-96 y_{n+\frac{3}{2}}+3 y_{n+\frac{1}{2}}=15 h_{n+\frac{5}{2}}+64 h_{n+2}+12 h f_{n+\frac{3}{2}}-h f_{n+\frac{1}{2}}$

Equations (2.6) are of uniform order 5, with error constant as follows

$[0.00488281250,0.00019531250,0.00019531250,0.02994791667,0.01666666667]^{\mathrm{T}}$

b) The second block scheme from $\mathrm{k}=3$ derived as follows

Equation (2.1) is interpolated at $x=x_{n+j}, j=0,1$ and equation (2.2) is collocated at $\mathbf{x}=\mathbf{x}_{n+j}, j=0, \frac{1}{2}, \frac{3}{2}, 2, \frac{5}{2}, 3$ which gives the system of non-linear equations of the form

$\sum_{\mathrm{j}=0}^{\mathrm{s}+\mathrm{t}-1} \alpha_{\mathrm{j}} \mathrm{x}_{\mathrm{n}+\mathrm{u}}^{\mathrm{j}}=\mathrm{y}_{\mathrm{n}+\mathrm{u}}, \mathrm{u}=0,1$

$\sum_{j=1}^{\mathrm{s}+\mathrm{t}-1} \mathrm{j} \alpha_{\mathrm{j}} \mathrm{x}_{\mathrm{n}+\mathrm{w}}^{\mathrm{j}-1}=\mathrm{f}_{\mathrm{n}+\mathrm{w}}, \mathrm{w}=0, \frac{1}{2}, \frac{3}{2}, 2, \frac{5}{2}, 3$

With the use of maple 17 mathematical software, we obtain the continuous formula for the method as

$\mathrm{y}(\mathrm{x})=\left[1+\frac{210(90)}{11 h^{2}}\left(x-x_{n}\right)^{2}-\frac{140(351)}{11 h^{3}}\left(x-x_{n}\right)^{3}+\frac{105(461)}{11 h^{4}}\left(x-x_{n}\right)^{4}-\frac{168(137)}{11 h^{5}}\left(x-x_{n}\right)^{5}+\frac{280(19)}{11 h^{6}}\left(x-x_{n}\right)^{6}-\right.$ $48011 h 7 x-x n 7 y n$

$+\left[-\frac{210(90)}{11 h^{2}}\left(x-x_{n}\right)^{2}+\frac{140(351)}{11 h^{3}}\left(x-x_{n}\right)^{3}-\frac{105(461)}{11 h^{4}}\left(x-x_{n}\right)^{4}+\frac{168(137)}{11 h^{5}}\left(x-x_{n}\right)^{5}-\frac{280(19)}{11 h^{6}}\left(x-x_{n}\right)^{6}+\right.$

$48011 h 7 x-x n 7 y n+1$

$+\left[\frac{2(106245)}{165 h}\left(x-x_{n}\right)^{2}-\frac{2(275907)}{165 h^{2}}\left(x-x_{n}\right)^{3}+\frac{2(54327)}{33 h^{3}}\left(x-x_{n}\right)^{4}-\frac{2(129121)}{165 h^{4}}\left(x-x_{n}\right)^{5}+\frac{8(7460)}{165 h^{5}}\left(x-x_{n}\right)^{6}-\right.$

$5384165 h 6 x-x n 7 f n+12$

$+\left[\left(x-x_{n}\right)+\frac{507699}{1980 h}\left(x-x_{n}\right)^{2}-\frac{1990013}{2970 h^{2}}\left(x-x_{n}\right)^{3}+\frac{261731}{396 h^{3}}\left(x-x_{n}\right)^{4}-\frac{2(77829)}{495 h^{4}}\left(x-x_{n}\right)^{5}+\frac{2(53987)}{1485 h^{5}}\left(x-x_{n}\right)^{6}-\right.$

$3248495 h 6 x-x n 7 f n$

$+\left[\frac{4(7635)}{99 h}\left(x-x_{n}\right)^{2}-\frac{4(59663)}{297 h^{2}}\left(x-x_{n}\right)^{3}+\frac{4(19643)}{99 h^{3}}\left(x-x_{n}\right)^{4}-\frac{4(9363)}{99 h^{4}}\left(x-x_{n}\right)^{5}+\frac{8(3253)}{297 h^{5}}\left(x-x_{n}\right)^{6}-\frac{784}{99 h^{6}}(x-\right.$ $x n 7 f n+32$

$+\left[-\frac{24705}{132 h}\left(x-x_{n}\right)^{2}+\frac{32199}{66 h^{2}}\left(x-x_{n}\right)^{3}-\frac{63693}{132 h^{3}}\left(x-x_{n}\right)^{4}+\frac{2(3803)}{33 h^{4}}\left(x-x_{n}\right)^{5}-\frac{2(883)}{33 h^{5}}\left(x-x_{n}\right)^{6}+\frac{160}{33 h^{6}}(x-\right.$

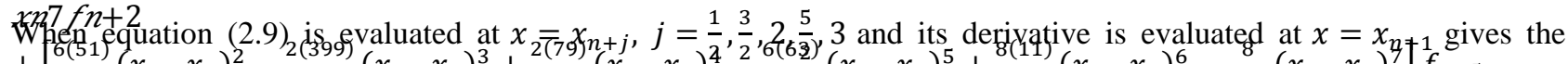

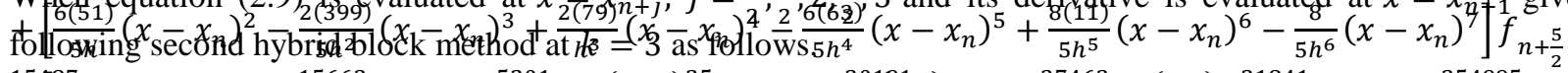

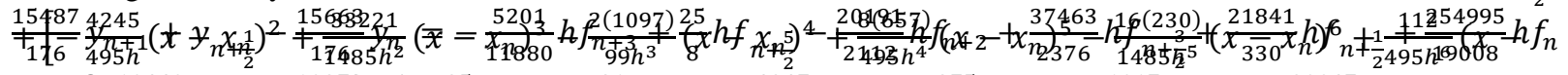
$\left.y_{n+\frac{3}{2}} n \frac{f n \mathbf{g}^{449}}{176} y_{n+1}+\frac{1027(32}{176} y_{n}^{9}\right)=\frac{25}{88} h f_{n+3}-\frac{81}{40} h f_{n+\frac{5}{2}}+\frac{4347}{704} h f_{n+2}-\frac{875}{88} h f_{n+\frac{3}{2}}-\frac{1917}{44} h f_{n+\frac{1}{2}}-\frac{30947}{3520} h f_{n}$ $y_{n+2}-\frac{512}{11} y_{n+1}+\frac{501}{11} y_{n}=\frac{332}{1485} h f_{n+3}-\frac{8}{5} h f_{n+\frac{5}{2}}+\frac{167}{33} h f_{n+2}-\frac{2192}{297} h f_{n+\frac{3}{2}}-\frac{5608}{165} h f_{n+\frac{1}{2}}-\frac{10189}{1485} h f_{n}$ $y_{n+\frac{5}{2}}-\frac{10625}{176} y_{n+1}+\frac{10449}{176} y_{n}=\frac{25}{88} h f_{n+3}-\frac{15}{8} h f_{n+\frac{5}{2}}+\frac{4875}{704} h f_{n+2}-\frac{875}{88} h f_{n+\frac{3}{2}}-\frac{975}{22} h f_{n+\frac{1}{2}}-\frac{6295}{704} h f_{n}$ $y_{n+3}-\frac{243}{11} y_{n+1}+\frac{232}{11} y_{n}=\frac{14}{55} h f_{n+3}+\frac{27}{11} h f_{n+2}-\frac{32}{11} h f_{n+\frac{3}{2}}-\frac{864}{55} h f_{n+\frac{1}{2}}-\frac{35}{11} h f_{n}$

$7560 y_{n+1}-7560 y_{n}=-37 h f_{n+3}+264 h f_{n+\frac{5}{2}}-807 h f_{n+2}+1328 h f_{n+\frac{3}{2}}+33 h f_{n+1}$

$+5640 h f_{n+\frac{1}{2}}+1139 h f_{n}$

Equations (2.10) are of uniform order 7, with error constant as follows.

$[0.002964268903,-0.001924034212,-0.001506132756,-0.001951936003,-0.007305194805$,

$-0.250000000000]^{T}$

c) The third block method at $\mathrm{k}=4$ derived as follows

Equation (2.1) is interpolated at $x=x_{n+j}, j=0, \frac{1}{2}$ and equation (2.2) is collocated at $x=x_{n+j}, j=0,1, \frac{3}{2}, 2, \frac{5}{2}$, $3, \frac{7}{2}, 4$ which gives the system of non-linear equations of the form

$\sum_{j=0}^{s+t-1} \alpha_{j} x_{n+i}^{j}=y_{n+i} \quad, i=0, \frac{1}{2}$

$\sum_{j=1}^{s+t-1} j \alpha_{j} x_{n+r}^{j-1}=f_{n+r} \quad, r=0,1, \frac{3}{2}, 2, \frac{5}{2}, 3, \frac{7}{2}, 4$

By applying the mathematical software to evaluate $\alpha_{j}$ in equations (2.11) and (2.12), we obtain the continuous formulation of the method as follows

$y(x)=\left[1-\frac{11520(2520)}{2233547 h^{2}}\left(x-x_{n}\right)^{2}+\frac{7680(8658)}{2233547 h^{3}}\left(x-x_{n}\right)^{3}-\frac{40320(1745)}{2233547 h^{4}}\left(x-x_{n}\right)^{4}+\frac{32256(1316)}{2233547 h^{5}}\left(x-x_{n}\right)^{5}-\right.$ $\left.\frac{26880(575)}{2233547 h^{6}}\left(x-x_{n}\right)^{6}+\frac{46080(73)}{2233547 h^{7}}\left(x-x_{n}\right)^{7}-\frac{11520(35)}{2233547 h^{8}}\left(x-x_{n}\right)^{8}+\frac{20480}{2233547 h^{9}}\left(x-x_{n}\right)^{9}\right] y_{n}+\left[\frac{11520(2520)}{2233547 h^{2}}\left(x-x_{n}\right)^{2}-\right.$ $\frac{7860(8658)}{2233547 h^{3}}\left(x-x_{n}\right)^{3}+\frac{40320(1745)}{2233547 h^{4}}\left(x-x_{n}\right)^{4}-\frac{32256(1316)}{2233547 h^{5}}\left(x-x_{n}\right)^{5}+\frac{26880(575)}{2233547 h^{6}}\left(x-x_{n}\right)^{6}-\frac{46080(73)}{2233547 h^{7}}\left(x-x_{n}\right)^{7}+$ 
$\left.\frac{11520(35)}{2233547 h^{8}}\left(x-x_{n}\right)^{8}-\frac{20480}{2233547 h^{9}}\left(x-x_{n}\right)^{9}\right] y_{n+\frac{1}{2}}+\left[\left(x-x_{n}\right)-\frac{26083334763}{5628538440 h}\left(x-x_{n}\right)^{2}+\frac{140072533945}{16885615320 h^{2}}\left(x-x_{n}\right)^{3}-\right.$ $\frac{12835932101}{1608153840 h^{3}}\left(x-x_{n}\right)^{4}+\frac{3685624841}{804076920 h^{4}}\left(x-x_{n}\right)^{5}-\frac{977094389}{603057690 h^{5}}\left(x-x_{n}\right)^{6}+\frac{243330815}{703567305 h^{6}}\left(x-x_{n}\right)^{7}-\frac{28776259}{703567305 h^{7}}(x-$ $\left.\left.x_{n}\right)^{8}+\frac{32166}{15634829 h^{8}}\left(x-x_{n}\right)^{9}\right] f_{n}+\left[-\frac{161926615320}{2814269220 h}\left(x-x_{n}\right)^{2}+\frac{95032968858}{4221403830 h^{2}}\left(x-x_{n}\right)^{3}-\frac{24922255031}{804076920 h^{3}}\left(x-x_{n}\right)^{4}+\right.$ $\frac{4405881735}{201019230 h^{4}}\left(x-x_{n}\right)^{5}-\frac{2674228355}{301528845 h^{5}}\left(x-x_{n}\right)^{6}+\frac{16(91437513)}{703567305 h^{6}}\left(x-x_{n}\right)^{7}-\frac{2(92605849)}{703567305 h^{7}}\left(x-x_{n}\right)^{8}+\frac{5882792}{422140383 h^{8}}(x-$ $\left.\left.x_{n}\right)^{9}\right] f_{n+1}+\left[\frac{8(760388160)}{703567305 h}\left(x-x_{n}\right)^{2}-\frac{8(9602705048)}{2110701915 h^{2}}\left(x-x_{n}\right)^{3}+\frac{4(1392410347)}{100509615 h^{3}}\left(x-x_{n}\right)^{4}-\frac{8(534221437)}{100509615 h^{4}}\left(x-x_{n}\right)^{5}+\right.$ $\frac{8(690939655)}{301528845 h^{5}}\left(x-x_{n}\right)^{6}-\frac{8(397082471)}{703567305 h^{6}}\left(x-x_{n}\right)^{7}+\frac{32(13063456)}{703567305 h^{7}}\left(x-x_{n}\right)^{8}-$

$\left.\frac{1521440}{46904487 h^{8}}\left(x-x_{n}\right)^{9}\right] f_{n+\frac{3}{2}}+\left[-\frac{1592185770}{187617948 h}\left(x-x_{n}\right)^{2}+\frac{6930177687}{187617948 h^{2}}\left(x-x_{n}\right)^{3}-\frac{1583999026}{26802564 h^{3}}\left(x-x_{n}\right)^{4}+\frac{1283634229}{26802564 h^{4}}(x-\right.$ $\left.\left.x_{n}\right)^{5}-\frac{2(72767514)}{6700641 h^{5}}\left(x-x_{n}\right)^{6}+\frac{2(131109257)}{46904487 h^{6}}\left(x-x_{n}\right)^{7}-\frac{4(8955226)}{46904487 h^{7}}\left(x-x_{n}\right)^{8}+\frac{2018012}{46904487 h^{8}}\left(x-x_{n}\right)^{9}\right] f_{n+2}$ $\left[\frac{8(489445992)}{703567305 h}\left(x-x_{n}\right)^{2}-\frac{16(3257587350)}{2110701915 h^{2}}\left(x-x_{n}\right)^{3}+\frac{4(1022387309)}{100509615 h^{3}}\left(x-x_{n}\right)^{4}-\frac{16(214704897)}{100509615 h^{4}}\left(x-x_{n}\right)^{5}+\right.$ $\left.\frac{8(607526579)}{301528845 h^{5}}\left(x-x_{n}\right)^{6}-\frac{16(189618885)}{703567305 h^{6}}\left(x-x_{n}\right)^{7}+\frac{32(13425587)}{703567305 h^{7}}\left(x-x_{n}\right)^{8}-\frac{14999104}{422140383 h^{8}}\left(x-x_{n}\right)^{9}\right] f_{n+\frac{5}{2}}$ $\left[-\frac{1325107560}{562853844 h}\left(x-x_{n}\right)^{2}+\frac{8930443094}{844280766 h^{2}}\left(x-x_{n}\right)^{3}-\frac{2857792229}{160815384 h^{3}}\left(x-x_{n}\right)^{4}+\frac{615336859}{40203846 h^{4}}\left(x-x_{n}\right)^{5}-\frac{448405193}{60305769 h^{5}}(x-\right.$ $\left.\left.x_{n}\right)^{6}+\frac{8(36160979)}{140713461 h^{6}}\left(x-x_{n}\right)^{7}-\frac{2(21192619)}{140713461 h^{7}}\left(x-x_{n}\right)^{8}+\frac{849992}{46904487 h^{8}}\left(x-x_{n}\right)^{9}\right] f_{n+3}+\left[\frac{8(17077320)}{234522435}\left(x-x_{n}\right)^{2}-\right.$ $\frac{8(11057796)}{33503205 h^{2}}\left(x-x_{n}\right)^{3}+\frac{8(18835348)}{33503205 h^{3}}\left(x-x_{n}\right)^{4}-\frac{8(16521835)}{33503205 h^{4}}\left(x-x_{n}\right)^{5}+\frac{8(8212230)}{33503205 h^{5}}\left(x-x_{n}\right)^{6}-\frac{8(16337317)}{234522435 h^{6}}\left(x-x_{n}\right)^{7}+$ $\left.\frac{32(617683)}{234522435 h^{7}}\left(x-x_{n}\right)^{8}-\frac{35168}{6700641 h^{8}}\left(x-x_{n}\right)^{9}\right] f_{n+\frac{7}{2}}+\quad+\left[-\frac{361321065}{5628538440 h}\left(x-x_{n}\right)^{2}+\frac{4945277457}{16885615320 h^{2}}\left(x-x_{n}\right)^{3}-\right.$ $\frac{810541603}{1608153840 h^{3}}\left(x-x_{n}\right)^{4}+\frac{360476553}{804076920 h^{4}}\left(x-x_{n}\right)^{5}-\frac{136825315}{603057690 h^{5}}\left(x-x_{n}\right)^{6}+\frac{46414443}{703567305 h^{6}}\left(x-x_{n}\right)^{7}-\frac{7223237}{703567305 h^{7}}(x-$ $\left.\left.x_{n}\right)^{8}+\frac{279698}{422140383 h^{8}}\left(x-x_{n}\right)^{9}\right] f_{n+4}$

When equation (2.13) is evaluated at $x=\left\{x_{n+1}, x_{n+\frac{3}{2}}, x_{n+2}, x_{n+\frac{5}{2}}, x_{n+3}, x_{n+\frac{7}{2}}, x_{n+4}\right\}$ and its derivative is evaluated at $x=\left\{x_{n+\frac{1}{2}}\right\}$, we have the following discrete block schemes at $k=4$ as follows.

$y_{n+1}-\frac{2921344}{2233547} y_{n+\frac{1}{2}}+\frac{687797}{2233547} y_{n} \quad=\frac{16521235}{6754246128} h f_{n+4}-\frac{5339968}{234522435} h f_{n+\frac{7}{2}}+\frac{321957235}{3377123064} h f_{n+3}-\frac{501362452}{2110701915} h f_{n+\frac{5}{2}}+$ $\frac{37060021}{93808974} h f_{n+2}-\frac{202157692}{422140383} h f_{n+\frac{3}{2}}+\frac{10848341641}{16885615320} h f_{n+1}-\frac{1691229383}{33771230640} h f_{n}$

$y_{n+\frac{3}{2}}-\frac{2871531}{2233547} y_{n+\frac{1}{2}}+\frac{637984}{2233547} y_{n} \quad=\frac{1185903}{625393160} h f_{n+4}-\frac{5458077}{312696580} h f_{n+\frac{7}{2}}+\frac{8979181}{125078632} h f_{n+3}-\frac{13527684}{78174145} h f_{n+\frac{5}{2}}+$ $\frac{33120711}{125078632} h f_{n+2}-\frac{309760791}{312696580} h f_{n+\frac{3}{2}}+\frac{534113739}{625393160} h f_{n+1}-\frac{14316959}{312696580} h f_{n}$

$y_{n+2}-\frac{2889728}{2233547} y_{n+\frac{1}{2}}+\frac{656181}{2233547} y_{n} \quad=\frac{356227}{156348290} h f_{n+4}-\frac{1668864}{78174145} h f_{n+\frac{7}{2}}+\frac{1417114}{15634829} h f_{n+3}-\frac{18389824}{78174145} h f_{n+\frac{5}{2}}+$ $\frac{9020556}{15634829} h f_{n+2}-\frac{12336704}{78174145} h f_{n+\frac{3}{2}}+\frac{64841534}{78174145} h f_{n+1}-\frac{7415927}{156348290} h f_{n} y_{n+\frac{5}{2}}-\frac{2876875}{2233547} y_{n+\frac{1}{2}}+\frac{643328}{2233547} y_{n}=\frac{762400}{422140383} h f_{n+4}-$ $\frac{754475}{46904487} h f_{n+\frac{7}{2}}+\frac{25265375}{422140383} h f_{n+3}+\frac{12835510}{422140383} h \mathrm{f}_{n+\frac{5}{2}}+\frac{41250875}{46904487} h f_{n+2} \quad-\frac{43726475}{422140383} h f_{n+\frac{3}{2}}+\frac{355948525}{422140383} h f_{n+1}-$ $\frac{19519615}{422140383} h f_{n}$

$y_{n+3}-\frac{2892672}{2233547} y_{n+\frac{1}{2}}+\frac{659125}{2233547} y_{n}=\frac{101655}{35736752} h f_{n+4}-\frac{470880}{15634829} h f_{n+\frac{7}{2}}+\frac{5125415}{17868376} h f_{n+3}+\frac{871020}{2233547} h f_{n+\frac{5}{2}}+\frac{3437775}{4467094} h f_{n+2}$ $+\frac{341620}{2233547} h f_{n+\frac{3}{2}}+\frac{14796945}{1786376} h f_{n+1}-\frac{11939465}{250157264} h f_{n}$

$y_{n+\frac{7}{2}}-\frac{2858219}{2233547} y_{n+\frac{1}{2}}+\frac{624672}{2233547} y_{n} \quad=-\frac{34055}{7868376} h f_{n+4}+\frac{7151193}{44670940} h f_{n+\frac{7}{2}}+\frac{13065115}{17868376} h f_{n+3}+\frac{1733032}{11167735} h f_{n+\frac{5}{2}}+$ $\frac{16803129}{17868376} h f_{n+2}+\frac{543263}{8934188} h f_{n+\frac{3}{2}}+\frac{76733069}{89341880} h f_{n+1}-\frac{1985543}{44670940} h f_{n}$

$y_{n+4}-\frac{3014656}{2233547} y_{n+\frac{1}{2}}+\frac{781109}{2233547} y_{n} \quad=\frac{43979726}{301528845} h f_{n+4}+\frac{25883648}{33503205} h f_{n+\frac{7}{2}}+\frac{6585152}{60305769} h f_{n+3}+\frac{270104576}{301528845} h f_{n+\frac{5}{2}}+$ $\frac{1979768}{6700641} h f_{n+2}+\frac{132772864}{301528845} h f_{n+\frac{3}{2}}+\frac{218727488}{301528845} h f_{n+1}-\frac{17926706}{301528845} h f_{n}$

$7257600 y_{n+\frac{1}{2}}-7257600 y_{n}$

$=-33953 h f_{n+4}+312874 f_{n+\frac{7}{2}}-1291214 h f_{n+3}+3146338 h f_{n+\frac{5}{2}}-5033120 h f_{n+2}+5595358 h f_{n+\frac{3}{2}}-$

$4604594 h f_{n+1}+4467094 h f_{n+\frac{1}{2}}+1070017 h f_{n}$

Equation (2.14) is of uniform order 9, with error constant as follows. [-0.000003803135250, -0.000003097181856, $0.000003495965842,-0.000003115623046, \quad-0.000003704193292, \quad-0.000002155631643, \quad-0.00001040304010, \quad-$ $55.938476562500000]^{T}$ 


\section{Block analysis of the methods}

The method in (2.6) is arranged in matrix form as

$$
\begin{aligned}
& \left(\begin{array}{ccccc}
\frac{225}{128} & 0 & -\frac{25}{16} & 0 & -\frac{153}{128} \\
-\frac{45}{128} & 1 & -\frac{9}{16} & 0 & -\frac{11}{128} \\
-\frac{11}{128} & 0 & -\frac{9}{16} & 1 & -\frac{45}{128} \\
915 & 0 & -480 & 0 & -435
\end{array}\right)\left(\begin{array}{l}
y_{n+\frac{1}{2}} \\
y_{n+1} \\
y_{n+\frac{3}{2}} \\
y_{n+2} \\
y_{n+\frac{5}{2}}
\end{array}\right)=\left(\begin{array}{ccccc}
0 & 0 & 0 & 0 & -1 \\
0 & 0 & 0 & 0 & 0 \\
0 & 0 & 0 & 0 & 0 \\
0 & 0 & 0 & 0 & 0 \\
0 & 0 & 0 & 0 & 0
\end{array}\right)\left(\begin{array}{c}
y_{n-2} \\
y_{n-\frac{3}{2}} \\
y_{n-1} \\
y_{n-\frac{1}{2}} \\
y_{n}
\end{array}\right) \\
& +\left(\begin{array}{ccccc}
-\frac{225}{128} & 0 & -\frac{75}{32} & 0 & -\frac{45}{128} \\
\frac{9}{128} & 0 & -\frac{9}{32} & 0 & -\frac{3}{128} \\
\frac{3}{128} & 0 & \frac{9}{32} & 0 & -\frac{9}{128} \\
-465 & 0 & -820 & 0 & -129 \\
-1 & 0 & 12 & 64 & 15
\end{array}\right)\left(\begin{array}{c}
f_{n+\frac{1}{2}} \\
f_{n+1} \\
f_{n+\frac{3}{2}} \\
f_{n+2} \\
f_{n+\frac{5}{2}}
\end{array}\right)+\left(\begin{array}{ccccc}
0 & 0 & 0 & 0 & 0 \\
0 & 0 & 0 & 0 & 0 \\
0 & 0 & 0 & 0 & 0 \\
0 & 0 & 0 & 0 & 64 \\
0 & 0 & 0 & 0 & 0
\end{array}\right)\left(\begin{array}{c}
f_{n-2} \\
f_{n-\frac{3}{2}} \\
f_{n-1} \\
f_{n-\frac{1}{2}} \\
f_{n}
\end{array}\right)
\end{aligned}
$$

Let

$$
\begin{aligned}
\mathrm{A}^{(0)} & =\left(\begin{array}{ccccc}
\frac{225}{128} & 0 & -\frac{25}{16} & 0 & -\frac{153}{128} \\
-\frac{45}{128} & 1 & -\frac{9}{16} & 0 & -\frac{11}{128} \\
-\frac{11}{128} & 0 & -\frac{9}{16} & 1 & -\frac{45}{128} \\
915 & 0 & -480 & 0 & -435 \\
3 & 0 & -96 & 0 & -93
\end{array}\right) \quad \mathrm{A}^{(1)}=\left(\begin{array}{ccccc}
0 & 0 & 0 & 0 & -1 \\
0 & 0 & 0 & 0 & 0 \\
0 & 0 & 0 & 0 & 0 \\
0 & 0 & 0 & 0 & 0 \\
0 & 0 & 0 & 0 & 0
\end{array}\right) \\
\mathrm{B}^{(0)} & =\left(\begin{array}{ccccc}
-\frac{225}{128} & 0 & -\frac{75}{32} & 0 & -\frac{45}{128} \\
\frac{9}{128} & 0 & -\frac{9}{32} & 0 & -\frac{3}{128} \\
\frac{3}{128} & 0 & \frac{9}{32} & 0 & -\frac{9}{128} \\
-465 & 0 & -820 & 0 & -129 \\
-1 & 0 & 12 & 64 & 15
\end{array}\right) \quad \mathrm{B}^{(1)}=\left(\begin{array}{ccccc}
0 & 0 & 0 & 0 & 0 \\
0 & 0 & 0 & 0 & 0 \\
0 & 0 & 0 & 0 & 0 \\
0 & 0 & 0 & 0 & 64 \\
0 & 0 & 0 & 0 & 0
\end{array}\right)
\end{aligned}
$$

We shall normalize the block method (3.1) by multiplying matrices $A^{(o)}, A^{(1)}, B^{(o)}, B^{(1)}$ with inverse of $A^{(o)}$ to obtain $A^{\prime(o)} \cdot A^{\prime(1)}, B^{\prime(o)}$ and $B^{\prime(1)}$

Then, $\rho(R)=\operatorname{det}\left[R \mathrm{~A}^{\prime(o)}-A^{\prime(1)}\right]$

$=\operatorname{det}\left[R\left(\begin{array}{ccccc}1 & 0 & 0 & 0 & 0 \\ 0 & 1 & 0 & 0 & 0 \\ 0 & 0 & 1 & 0 & 0 \\ 0 & 0 & 0 & 1 & 0 \\ 0 & 0 & 0 & 0 & 1\end{array}\right)-\left(\begin{array}{ccccc}0 & 0 & 0 & 0 & 1 \\ 0 & 0 & 0 & 0 & 1 \\ 0 & 0 & 0 & 0 & 1 \\ 0 & 0 & 0 & 0 & 1 \\ 0 & 0 & 0 & 0 & 1\end{array}\right)\right]=0$

$=\operatorname{det}\left[\left(\begin{array}{ccccc}\mathrm{R} & 0 & 0 & 0 & -1 \\ 0 & \mathrm{R} & 0 & 0 & -1 \\ 0 & 0 & \mathrm{R} & 0 & -1 \\ 0 & 0 & 0 & \mathrm{R} & -1 \\ 0 & 0 & 0 & 0 & \mathrm{R}-1\end{array}\right)\right]=\mathrm{R}^{5}-\mathrm{R}^{4}=0$

Which implies that $\mathrm{R}_{1}=\mathrm{R}_{2}=\mathrm{R}_{3}=\mathrm{R}_{4}=0$ and $\mathrm{R}_{5}=1$. Hence from the definition (1.3) equation(3.2), the method (3.1) is zero stable and also consistent as its order is $[5,5,5,5,5]^{\mathrm{T}}>1$, thus convergent.

The same analysis holds for block methods (2.10) and (2.12), thus they are zero stable and convergent

\section{Numerical experiments}

The two block methods derived at $\mathrm{k}=3$ and one block method at $\mathrm{k}=4$ are demonstrated with the following problems Problem 1

$\mathrm{y}^{\prime}=-\mathrm{y} \quad \mathrm{y}(0)=1, \quad 0 \leq \mathrm{x} \leq 2, \quad \mathrm{~h}=0.1$

Exact solution: $y(x)=e^{-x}$

Problem 2

$\mathrm{y}^{\prime}=\mathrm{xy} \quad \mathrm{y}(0)=1, \mathrm{~h}=0.1$

Exact solution: $y(x)=e^{\frac{x^{2}}{2}}$

Problem 3

$\mathrm{y}^{\prime}+4 \mathrm{y}=20 \quad \mathrm{y}(0)=2 \quad \mathrm{~h}=0.01$

Exact solution: $\mathrm{y}(\mathrm{x})=5-3 \mathrm{e}^{-4 \mathrm{x}}$ 
Table 1: Approximate Solution to Problem 1 from Odekunle Et Al [7]

\begin{tabular}{|c|c|c|c|c|}
\hline $\mathrm{X}$ & Exact solution & EOAS (1) & EOAS (2) & EMY \\
\hline 0.1000 & 0.9048374180359595 & $1.5490(-08)$ & $2.3231(-07)$ & $2.5292(-06)$ \\
\hline 0.2000 & 0.8187307530779818 & $9.3743(-09)$ & $1.0067(-07)$ & $2.0937(-06)$ \\
\hline 0.3000 & 0.7408182206817178 & $1.6932(-08)$ & $3.2505(-07)$ & $2.0079(-06)$ \\
\hline 0.4000 & 0.6703200460356393 & $1.6406(-08)$ & $4.6622(-07)$ & $1.6198(-06)$ \\
\hline 0.5000 & 0.6065306597126334 & $2.5228(-08)$ & $3.4071(-07)$ & $3.1608(-06)$ \\
\hline 0.6000 & 0.5488116360940264 & $1.9716(-08)$ & $4.8161(-07)$ & $2.7294(-06)$ \\
\hline 0.7000 & 0.4965853037914095 & $2.3504(-08)$ & $5.6328(-07)$ & $2.5457(-06)$ \\
\hline 0.8000 & 0.4493289641172216 & $2.1995(-08)$ & $4.4956(-07)$ & $2.1713(-06)$ \\
\hline 0.9000 & 0.4065696597405991 & $2.6862(-08)$ & $5.3518(-07)$ & $3.1008(-06)$ \\
\hline 1.0000 & 0.3678794411714423 & $2.2220(-08)$ & $5.7870(-07)$ & $2.7182(-06)$ \\
\hline
\end{tabular}

Table 2: Approximate Solution to Problem 1 with New Block Methods Derived

\begin{tabular}{ccccc}
\multicolumn{6}{c}{ Table 2: Approximate Solution to Problem 1 with New Block Methods Derived } \\
\hline $\mathrm{x}$ & Exact solution & Method A $(\mathrm{k}=3)$ & Method B $(\mathrm{k}=3)$ & Method C $(\mathrm{k}=4)$ \\
\hline 0.1000 & 0.9048374180359595 & 0.9048374180359595 & 0.904837418035702 & 0.904837418035966 \\
0.2000 & 0.8187307530779818 & 0.8187307530779818 & 0.818730753077778 & 0.818730753077968 \\
0.3000 & 0.7408182206817178 & 0.7408182206817178 & 0.740818220681792 & 0.740818220681725 \\
0.4000 & 0.6703200460356393 & 0.6703200460356393 & 0.670320046041488 & 0.670320046035640 \\
0.5000 & 0.6065306597126334 & 0.6065306597126334 & 0.606530659699514 & 0.606530659712627 \\
0.6000 & 0.5488116360940264 & 0.5488116360940264 & 0.548811636184434 & 0.548811636094037 \\
0.7000 & 0.4965853037914095 & 0.4965853037914095 & 0.496585303377540 & 0.496585303791423 \\
0.8000 & 0.4493289641172216 & 0.4493289641172216 & 0.449328966219365 & 0.449328964117217 \\
0.9000 & 0.4065696597405991 & 0.4065696597405991 & 0.406569649330592 & 0.406569659740614 \\
1.0000 & 0.3678794411714423 & 0.3678794411714423 & 0.367879493036357 & 0.367879441171460 \\
\hline
\end{tabular}

Table 3: Absolute Error of Problem 1

\begin{tabular}{cccc}
\hline $\mathrm{X}$ & Error of Method A at k=3 & Error of Method B at k=3 & Error of Method C at k= 4 \\
\hline 0.1000 & $2.5198(-10)$ & $2.5800(-13)$ & $6.0000(-15)$ \\
0.2000 & $6.9206(-10)$ & $2.0400(-13)$ & $1.4000(-14)$ \\
0.3000 & $7.3009(-11)$ & $7.4000(-14)$ & $7.0000(-15)$ \\
0.4000 & $1.7709(-9)$ & $5.8490(-12)$ & $1.0000(-15)$ \\
0.5000 & $4.2900(-9)$ & $1.3119(-11)$ & $6.0000(-15)$ \\
0.6000 & $1.4894(-8)$ & $9.0408(-11)$ & $1.1000(-14)$ \\
0.7000 & $4.6803(-8)$ & $4.1387(-10)$ & $1.3000(-14)$ \\
0.8000 & $1.5088(-7)$ & $2.1021(-9)$ & $5.0000(-15)$ \\
0.9000 & $4.8319(-7)$ & $1.0410(-8)$ & $1.5000(-14)$ \\
1.0000 & $1.5500(-6)$ & $5.1865(-8)$ & $1.8000(-14)$ \\
\hline
\end{tabular}

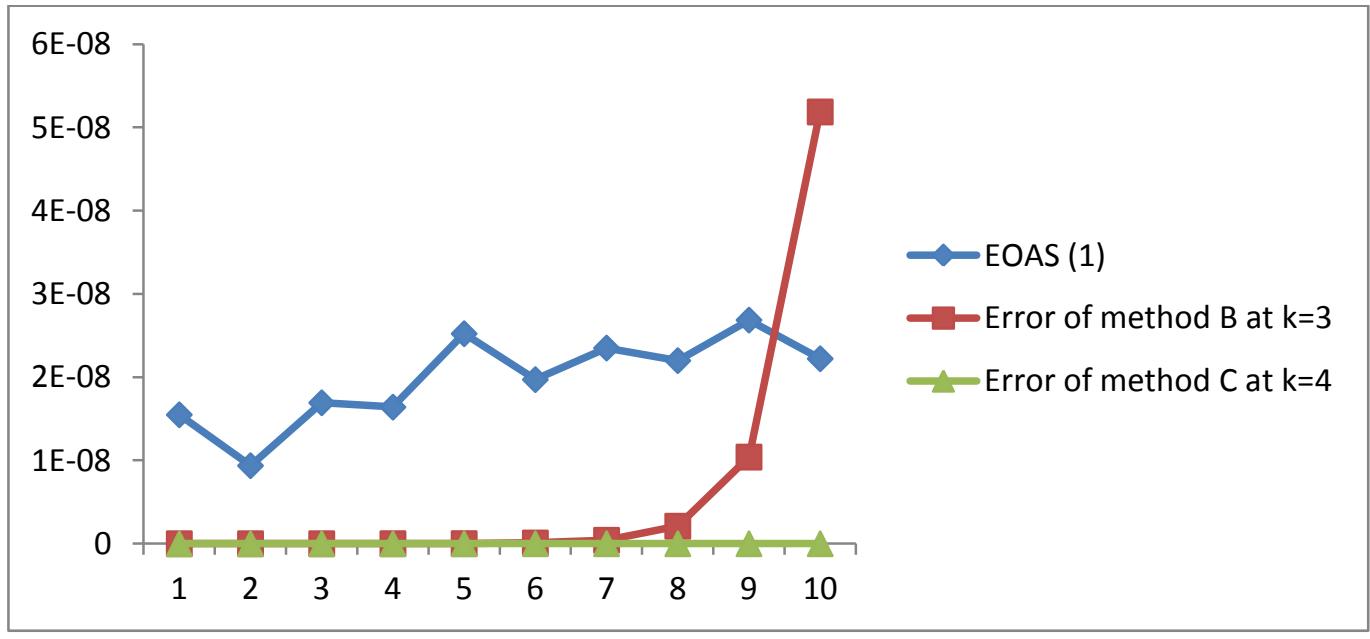

Fig. 1: Error Graph of Problem1 


\begin{tabular}{ccccc}
\hline $\mathrm{x}$ & Exact solution & EOAS (1) & EOAS (2) & EBM \\
\hline 0.1000 & 1.0050125208594010 & $3.1386(-07)$ & $5.2398(-07)$ & $5.29(-07)$ \\
0.2000 & 1.0202013400267558 & $1.3364(-07)$ & $1.6913(-07)$ & $1.77(-07)$ \\
0.3000 & 1.0460278599087169 & $3.1819(-07)$ & $8.7243(-07)$ & $8.99(-07)$ \\
0.4000 & 1.0832870676749586 & $9.8972(-09)$ & $3.0098(-06)$ & $3.09(-06)$ \\
0.5000 & 1.1331484530668263 & $6.9521(-07)$ & $1.7466(-06)$ & $1.91(-06)$ \\
0.6000 & 1.1972173631218102 & $4.0794(-07)$ & $4.1710(-06)$ & $4.48(-06)$ \\
0.7000 & 1.2776213132048868 & $7.7319(-07)$ & $9.6465(-06)$ & $1.02(-05)$ \\
0.8000 & 1.3771277643359572 & $7.0821(-07)$ & $6.7989(-06)$ & $7.74(-05)$ \\
0.9000 & 1.4993025000567668 & $2.8682(-06)$ & $1.2913(-05)$ & $1.44(-05)$ \\
1.0000 & 1.6487212707001282 & $2.0664(-06)$ & $2.6575(-05)$ & $2.93(-05)$ \\
\hline
\end{tabular}

Table 5: Approximate Solution to Problem 2 at $\mathrm{H}=0.1$ with the New Block Methods Derived

\begin{tabular}{ccccc}
\hline $\mathrm{x}$ & Exact solution & Method A $(\mathrm{k}=3)$ & Method A $(\mathrm{k}=3)$ & $\mathrm{Method} \mathrm{C}(\mathrm{k}=4)$ \\
\hline 0.1000 & 1.0050125208594010 & 1.005012524786800 & 1.005012520821450 & 1.005012520858670 \\
0.2000 & 1.0202013400267558 & 1.020201353563710 & 1.020201339988210 & 1.020201340026000 \\
0.3000 & 1.0460278599087169 & 1.046027864154210 & 1.046027859908610 & 1.046027859907970 \\
0.4000 & 1.0832870676749586 & 1.083287098480390 & 1.083287068619520 & 1.083287067674950 \\
0.5000 & 1.1331484530668263 & 1.133148409900560 & 1.133148451587550 & 1.133148453059620 \\
0.6000 & 1.1972173631218102 & 1.197217554200290 & 1.197217376385660 & 1.197217363191800 \\
0.7000 & 1.2776213132048868 & 1.277620800696280 & 1.277621259685030 & 1.277621312469230 \\
0.8000 & 1.3771277643359572 & 1.377129412581450 & 1.377128039667030 & 1.377127764031650 \\
0.9000 & 1.4993025000567668 & 1.499297604095880 & 1.499301198635990 & 1.499302579518210 \\
1.0000 & 1.6487212707001282 & 1.648736250960000 & 1.648727572198520 & 1.648719757723030 \\
\hline
\end{tabular}

Table 6: Absolute Error of Problem 2

\begin{tabular}{cccc}
\hline $\mathrm{X}$ & Error of Method A at $\mathrm{k}=3$ & Error of Method B at k=3 & Error of Method C at $\mathrm{k}=4$ \\
\hline 0.1000 & $3.9274(-9)$ & $3.7950(-11)$ & $7.3000(-13)$ \\
0.2000 & $1.3537(-8)$ & $3.8550(-11)$ & $7.6000(-13)$ \\
0.3000 & $4.2455(-9)$ & $1.1000(-13)$ & $7.5000(-13)$ \\
0.4000 & $3.0805(-8)$ & $9.4456(-10)$ & $1.0000(-14)$ \\
0.5000 & $4.3166(-8)$ & $1.4793(-9)$ & $7.2100(-12)$ \\
0.6000 & $1.9108(-7)$ & $1.3264(-8)$ & $6.9990(-11)$ \\
0.7000 & $5.1251(-7)$ & $5.3520(-8)$ & $7.3566(-10)$ \\
0.8000 & $1.6482(-6)$ & $2.7533(-7)$ & $3.0431(-10)$ \\
0.9000 & $4.8960(-6)$ & $1.3014(-6)$ & $7.9461(-8)$ \\
1.0000 & $1.4980(-5)$ & $6.3015(-6)$ & $1.5130(-6)$ \\
\hline
\end{tabular}

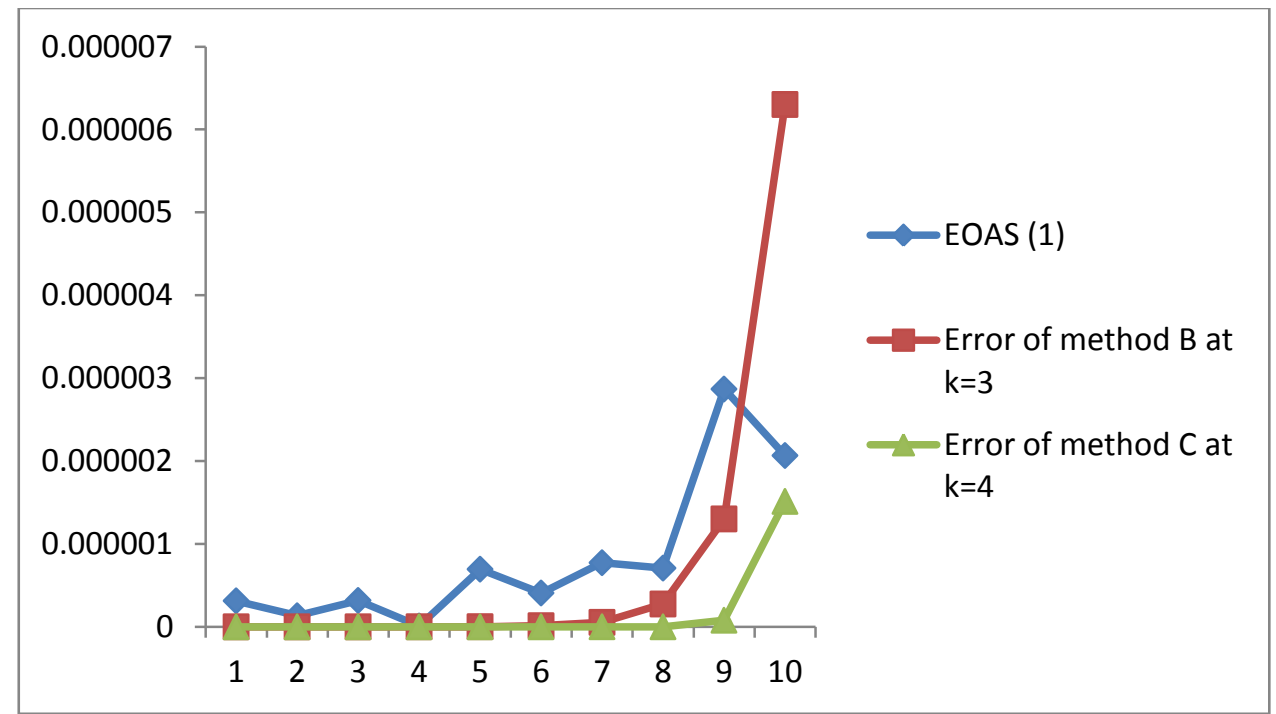

Fig. 2: Error Graph of Problem2 


\begin{tabular}{ccccc}
\hline $\mathrm{X}$ & Exact solution & Method A k =3 & Method B k =3 & Method C k =4 \\
\hline 0.01 & 2.117631682543030 & 2.117631682539970 & 2.117631682543050 & 2.11763168254302 \\
0.02 & 2.230650960840090 & 2.230650960830580 & 2.230650960840060 & 2.23065096084009 \\
0.03 & 2.339238689848530 & 2.33923868984853 & 2.339238689848450 & 2.33923868984851 \\
0.04 & 2.443568633101370 & 2.44356863310137 & 2.443568633100170 & 2.44356863310137 \\
0.05 & 2.543807740766050 & 2.54380774076605 & 2.543807740765050 & 2.543807740766560 \\
0.06 & 2.640116416800340 & 2.64011641680034 & 2.640116416773370 & 2.640116416796300 \\
0.07 & 2.732648775632820 & 2.73264877563282 & 2.732648775627240 & 2.732648775665830 \\
0.08 & 2.821552888778930 & 2.82155288877893 & 2.821552888157530 & 2.821552888218170 \\
0.09 & 2.906971021786910 & 2.90697102178691 & 2.906971022057390 & 2.906971029253120 \\
0.10 & 2.989039861893080 & 2.98903986189308 & 2.989039847321860 & 2.989039776916520 \\
\hline
\end{tabular}

Table 8: Absolute Error of Problem 3

\begin{tabular}{cccc}
\multicolumn{5}{c}{ Table 8: Absolute Error of Problem 3 } \\
\hline $\mathrm{x}$ & ERROR 0f Method A & ERROR 0f Method B & ERROR 0f Method C \\
\hline 0.01 & $3.0600(-12)$ & $2.0000(-14)$ & $1.1000(-14)$ \\
0.02 & $9.5100(-12)$ & $3.0000(-14)$ & - \\
0.03 & $6.3000(-13)$ & $8.0000(-14)$ & $2.1000(-14)$ \\
0.04 & $2.2760(-11)$ & $1.2000(-12)$ & - \\
0.05 & $4.7450(-11)$ & $1.0000(-12)$ & $5.1000(-13)$ \\
0.06 & $1.7270(-10)$ & $2.6970(-11)$ & $4.0400(-12)$ \\
0.07 & $5.2519(-10)$ & $5.5800(-12)$ & $3.3010(-11)$ \\
0.08 & $1.6816(-9)$ & $6.2140(-10)$ & $5.6076(-10)$ \\
0.09 & $5.3022(-9)$ & $2.7048(-10)$ & $7.4662(-9)$ \\
0.10 & $1.6794(-8)$ & $1.4571(-8)$ & $8.4977(-8)$ \\
\hline
\end{tabular}

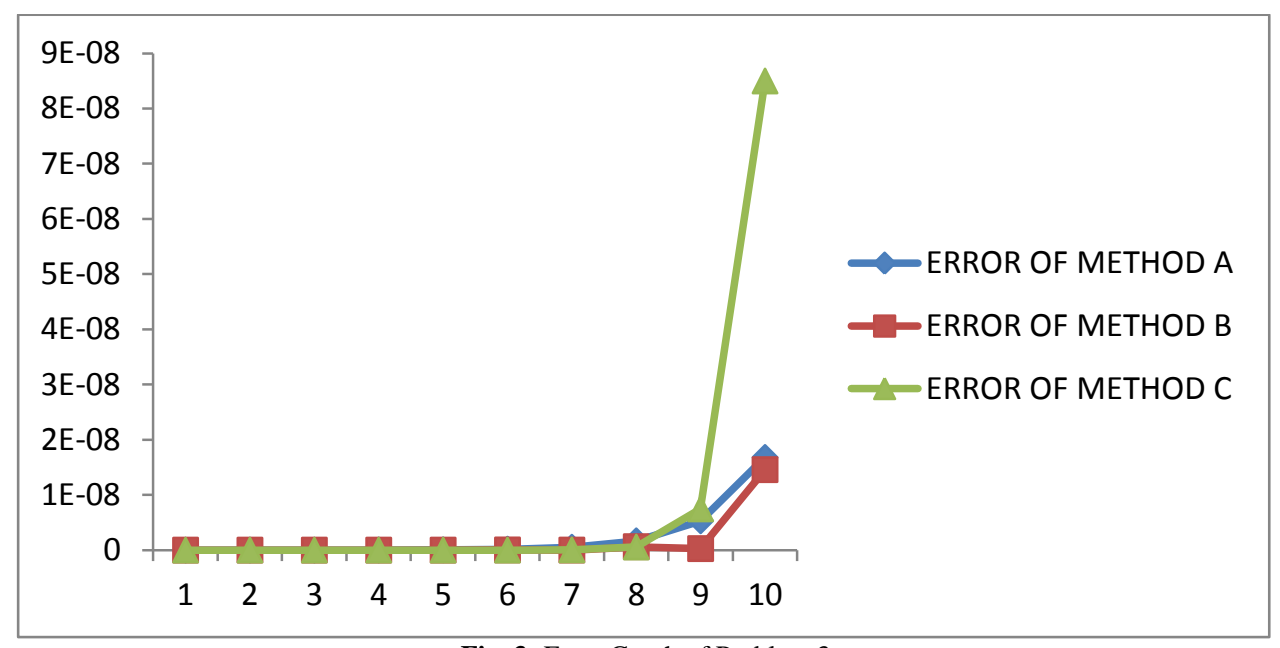

Fig. 3: Error Graph of Problem 3

\section{Discussion of results}

We observed that for the first two problems tested with our new block methods of (2.6) which is method A, (2.10) which is method B while (2.12) which is method C, the block method (2.12) of $\mathrm{k}=4$ shows it superiority but for the third problem the block method (2.10) performed best. (See Tables 1, 2, 3 and fig 1,2 and 3)

\section{Conclusion}

We want to draw our conclusion that the block of (2.12) of uniform order 9 at $\mathrm{k}=4$ and the block of (2.10) of uniform order 7 at $\mathrm{k}=3$ are suitable for the solution of (1.0). Also each of the new block method display its superiority over Odekunle e tal [7], [8] see error graphs of 1,2 and 3. 


\section{References}

[1] DO Awoyemi. A class of Continuous methods for general second order initial value problems in ordinary differential equations. International Journal of computer Mathematics. Volume72. (1999) pages 29-33 http://dx.doi.org/10.1080/00207169908804832.

[2] AM Badmus and DW Mshelia. Some uniform order block method for the solution of first-order ordinary differential equations. Journal of Nigerian Association of Mathematical Physics, 19, (2011), 149-154.

[3] AM Badmus and DW Mshelia Uniform order zero-stable k step methods for initial value problems of ordinary differential equations. Journal of Nigerian Association of Mathematical Physics volume 20, (2012) 65-74.

[4] AM Badmus and ZA Adegboye .Comparison of two new Quade's type hybrid Block methods for solution of ordinary differential equations. Journal of research in Physical Sciences, volume 6 No1 (2010) pages 71-76

[5] AM Badmus, TA Badmos and FE Ekpenyong. Hybrid Implicit Block algorithms for improved performances in the solution of first order initial value problems. Journal of Nigerian Association of Mathematical Physics volume 23, (2013) pages 95-102

[6] JD Lamber Computational Methods in Ordinary Differential Equations. John Wiley and Sons, New York. (1973) 278

[7] MR Odekunle, AO Adesanya and J Sunday. Four point block method for direct integration of first order ordinary differential equations. International Journal of Engineering Research and Applications (IJERA) ISSN: 2248-9622 Vol. 2, (2012a) pp.1182-1187.

[8] MR Odekunle, AO Adesanya and J Sunday. A new block integrator for the solution of initial value problems of first- order ordinary differential equations. International Journal of Pure and Applied Science and Technology, 11(2), (2012b), 92-100.

[9] AO Subair. A 3- and 4-Step Collocation Hybrid Block Method for Solution of First Order Ordinary Differential Equations". MSc thesis (unpublished) Nigeria Defence Academy Kaduna. (2014),

[10] YA Yahaya. And ZA Adegboye. A New Quade's Type 4-step blocks Hybrid Multistep Method for Accurate and Efficient Parallel Solution of Ordinary Differential Equations. Abacus 34, No 2B: (2007) 271-278. 